\title{
EXFOR - a global experimental nuclear reaction data repository: Status and new developments
}

\author{
Valentina Semkova ${ }^{1}$, Naohiko Otuka $^{1}$,, Marina Mikhailiukova ${ }^{2}$, Boris Pritychenko ${ }^{3}$, and Oscar Cabellos ${ }^{4}$, for the \\ International Network of Nuclear Reaction Data Centres (NRDC) \\ ${ }^{1}$ International Atomic Energy Agency, Nuclear Data Section, 1400 Vienna, Austria \\ 2 Institute of Physics and Power Engineering, Russian Nuclear Data Centre, 249033 Obninsk, Russia \\ 3 Brookhaven National Laboratory, National Nuclear Data Center, Upton NY 11973, USA \\ ${ }^{4}$ OECD NEA Data Bank, 92100 Boulogne-Billancourt, France
}

\begin{abstract}
Members of the International Network of Nuclear Reaction Data Centres (NRDC) have collaborated since the 1960s on the worldwide collection, compilation and dissemination of experimental nuclear reaction data. New publications are systematically complied, and all agreed data assembled and incorporated within the EXFOR database. Recent upgrades to achieve greater completeness of the contents are described, along with reviews and adjustments of the compilation rules for specific types of data.
\end{abstract}

\section{Introduction}

The EXFOR database [1] is a global repository of experimental nuclear reaction data and associated information that has been systematically collected, expanded and developed as a product of the international collaboration of Nuclear Reaction Data Centres (NRDC) under the auspices of the International Atomic Energy Agency Nuclear Data Section (IAEA NDS) since 1970. The efforts of the NRDC community to provide completeness and the high quality of the compilations made the EXFOR system a valuable source of detailed information for evaluations and analysis. This paper represents a short summary of the developments carried out since our previous report in 2014.

\section{NRDC network}

The current members of the network are listed in Table 1. CAJaD (Moscow) discontinued its activity in 2014, and its responsibility has been taken over by CNPD.
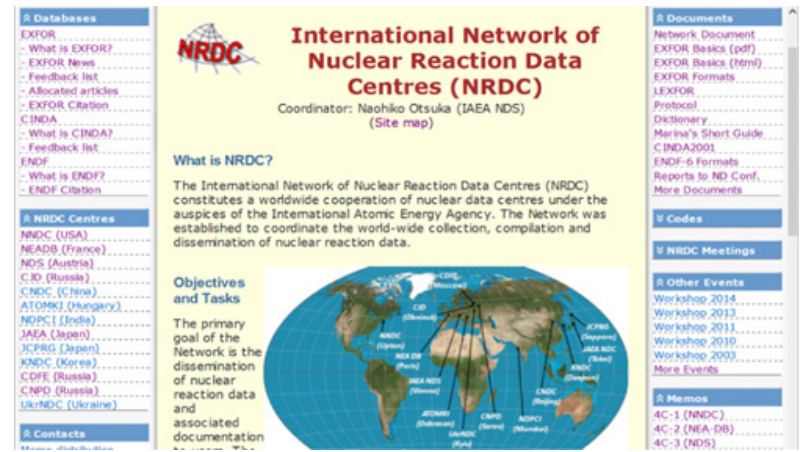

Figure 1. NRDC web page (http://www-nds.iaea.org/ $\mathrm{nrdc} /)$.

a e-mail: n.otsuka@iaea.org
Table 1. Agreed areas of responsibility of the NRDC centres (ND: neutron data; CPND: charged-particle data; PhND: photonuclear data).

\begin{tabular}{ll}
\hline Centre & Area of responsibility \\
\hline ATOMKI (Denbrecen) & CPND measured by ATOMKI \\
\hline CDFE (Moscow) & PhND from former USSR \\
\hline CJD (Obninsk) & ND from former USSR \\
\hline CNDC (Beijing) & ND+CPND of China \\
\hline CNPD (Sarov) & CPND from former USSR \\
\hline JAEA (Tokai) & Dissemination of JENDL \\
\hline JCPRG (Sapporo) & CPND+PhND from Japan \\
\hline KNDC (Dajeon) & All data from Korea \\
\hline NDPCI (Mumbai) & All data from India \\
\hline NDS (Vienna) & Data not covered by others \\
\hline NEA-DB (Paris) & ND+CNPD from DB countries \\
\hline NNDC (Upton) & All data from US+Canada \\
\hline UkrNDC (Kyiv) & All data from Ukraine \\
\hline
\end{tabular}

Compilers from Kazakhstan and Mongolia also contribute through compilation of experimental data measured in Kazakhstan and heavy-ion induced reaction data measured in West European countries, respectively. The NDS, NEA-DB and CJD peer review the preliminary EXFOR entries regularly before their inclusion in the EXFOR database.

IAEA NDS is responsible for the coordination of the network activities and for ensuring that the data compilation and exchange are undertaken in an effective, efficient and timely manner. The agreed organisational role of the NDS involves the following:

- Assign clear responsibilities to each centre, and drive these activities forward.

- Monitor progress in compilation and corrections.

- Decide on all issues relating to compilation rules and dictionary codes.

(C) The Authors, published by EDP Sciences. This is an Open Access article distributed under the terms of the Creative Commons Attribution License 4.0 (http://creativecommons.org/licenses/by/4.0/). 
- Update EXFOR and CINDA Master Files and Dictionaries.

- Organise NRDC meetings and EXFOR compilation workshops.

Various technical issues are discussed at the annual NRDC meetings (the last one was held in Beijing [2]). Meetings summaries, decisions and assigned actions are published as INDC(NDS) reports. Other reports, working papers and presentations (since 1999), compilation tools, and various NRDC-related materials are available from the NRDC web page (Fig. 1).

\section{EXFOR upgrades}

The scope of the EXFOR database has gradually expanded in response to the needs of new developments and applications. In this respect retroactive scanning of the scientific literature is required to provide completeness of the database for all types of data considered for compilation. Such checking of EXFOR coverage is periodically performed by NRDC members based on reference citations in review articles, nuclear data compilations, books etc., or user feedback. Subsequently, the data are analysed and compiled as a new EXFOR entry, or publications are added as additional references if the experimental work has already been compiled as an EXFOR entry. The systematic assessments of the EXFOR coverage have been recently carried out for the following specific types of data or areas of applications.

\subsection{Thermal neutron constants}

NDS staff have extracted all articles (excluding theses and private communications) related to experimental crosssections or resonance integrals (3144 articles) from the citation lists of "Atlas of Neutron Resonances" (2006) [3]. The list was sent to other centres for compilation. The same checking is ongoing at NNDC and NEA-DB. NDS also checked the 167 experimental thermal neutron constants (cross-sections and fission neutron multiplicities of ${ }^{233,235} \mathrm{U},{ }^{239,241} \mathrm{Pu}$ and ${ }^{252} \mathrm{Cf}$ ) assembled by Axton for his evaluation [4] against EXFOR during the revision of the IAEA neutron standard. See Ref. [5] for more details.

\subsection{Neutron dosimetry}

Availability of all measurements and the precise description of the experimental details for the monitor and dosimetry reactions are of particular importance. The dosimetry reaction cross-sections are employed for the determination and monitoring of neutron fluence and spectra at different facilities as well as for assessments of operational safety. An on-going IAEA coordinated research project (CRP) on "Testing and Improving the International Reactor Dosimetry and Fusion File (IRDFF)" is providing an updated library in the energy range up to $60 \mathrm{MeV}$ [6]. The EXFOR database has been assessed for:

- Coverage of articles published in all proceedings of the International Symposium on Reactor Dosimetry (ISRD) published between 1977 and 2012. A total of 41 articles were assigned for compilation in new Entries or for addition to existing Entries.

- Availability of neutron spectra employed in spectrum averaged cross-section measurements. Links to the source spectra compiled in IRDF-2002 have been provided in the EXFOR entries for the spectrumaveraged cross-sections measured at Intermediateenergy Standard Neutron Field (ISNF) in Gaithersburg, the Coupled Fast Reactivity Measuring Facility (CFRMF) in Idaho, the thermal-fast coupled field $(\Sigma-\Sigma)$ in Belgium, UK and Romania, and the central cavity in the fast research reactor YAYOI in Tokai.

- Neutron spectra for the charged-particle induced neutron source reactions, such as ${ }^{7} \mathrm{Li}(\mathrm{p}, \mathrm{n}+\mathrm{x}),{ }^{7} \mathrm{Li}(\mathrm{d}, \mathrm{n}+\mathrm{x})$ and ${ }^{9} \mathrm{Be}(\mathrm{d}, \mathrm{n}+\mathrm{x})$. A total of 207 data sets for total, differential, double differential cross-sections and neutron production yields of light-ion induced reactions on isotopes of $\mathrm{H}, \mathrm{D}, \mathrm{Li}, \mathrm{Be}, \mathrm{C}, \mathrm{Cu}, \mathrm{Mo}$, $\mathrm{Ta}, \mathrm{Au}, \mathrm{Fe}, \mathrm{Co}, \mathrm{Ni}, \mathrm{Nb}, \mathrm{Pb}, \mathrm{Pd}, \mathrm{Ag}, \mathrm{Pt}, \mathrm{Al}, \mathrm{W}$, water etc. were analyzed. Existing compilations were systematically improved and missing publications were assigned for compilations.

\subsection{Thermonuclear reaction rates for charged-particle-induced reactions}

Nuclear reaction data for projectiles with $\mathrm{A} \leq 12$ are compulsory for EXFOR compilation. The Nuclear Astrophysics Compilation of Reactions NACRE II [7] contains thermonuclear reaction rates for 34 chargedparticle induced reactions on nuclides with mass number $\mathrm{A}<16$. Contents of the EXFOR database were compared with the experimental data sets considered in the NACRE II evaluations. We concluded that more than $80 \%$ of the relevant articles are compiled in EXFOR. About 30 missing articles have been assigned for compilation by Centres according to the distribution of the compilation responsibilities.

\subsection{Prompt fission neutrons for non-destructive assay of nuclear materials}

Both fission neutron multiplicity distributions $\mathrm{P}(v)$ and average multiplicity ( $\nu$-bar) are important for the development of the passive non-destructive assay of nuclear materials. However, $\mathrm{P}(v)$ data had not been considered for EXFOR compilation until the necessary introduction of an EXFOR quantity code (NUM) in 2004. The EXFOR coverage of the prompt fission neutron multiplicity distribution and average multiplicity was checked against experimental works cited in the Holden evaluation [8]. As a consequence of this exercise, both $\mathrm{P}(v)$ and $\nu$-bar values of 11 experimental studies have been additionally compiled in EXFOR.

\subsection{Neutron reaction data for the CIELO project}

Compilations of data for ${ }^{1} \mathrm{H},{ }^{16} \mathrm{O},{ }^{56} \mathrm{Fe},{ }^{235} \mathrm{U},{ }^{238} \mathrm{U}$ and ${ }^{239} \mathrm{Pu}$ in EXFOR were revisited in connection with the evaluation work carried out by the Collaborative International Evaluated Library Organization (CIELO) [9]. The status of the database was improved by (1) collection of new data such as ${ }^{16} \mathrm{O}(\mathrm{n}, \alpha)^{13} \mathrm{C}$ crosssections measured at IPPE by Khryachkov (Entry 41575.002) and at IRMM by Georginis (Entry 23040.003); (2) replacement of digitised values with the original experimental data for the ${ }^{13} \mathrm{C}(\alpha, \mathrm{n}){ }^{16} \mathrm{O}$ cross-section measured at ORNL by Bair and $\operatorname{Haas}^{13} \mathrm{C}(\alpha, \mathrm{n}){ }^{16} \mathrm{O}$ (Entry C0489.002); (3) addition of digitised values 
in the old EXFOR entries compiled without data. A functionality for the conversion and plotting of the data for reversible nuclear reactions (for example ${ }^{13} \mathrm{C}\left(\alpha, \mathrm{n}_{0}\right){ }^{16} \mathrm{O}$ reaction cross-section into $\left.{ }^{16} \mathrm{O}\left(\mathrm{n}, \alpha_{0}\right){ }^{13} \mathrm{C}\right)$ by employing the principle of detailed balance has been developed by Zerkin at NDS and made available on the NDS EXFOR web retrieval system.

\subsection{Experimental kerma factors}

The NRDC network has been advised to compile kerma factor data as well as gas production cross-sections data needed for the validation of the radiation damage metrics. This recommendation arose from an IAEA CRP on "Primary Radiation Damage Cross-Sections" and a Technical Meeting on Nuclear Reaction Data and Uncertainties for Radiation Damage, as organised by NDS staff in 2013-2016 [10].

\section{Improvements of the quality and consistency of the EXFOR compilation}

Over the course of several decades of EXFOR development, the basic principles and the format have proven to be a robust and efficient way of performing data storage and retrieval. Many new types of data have been introduced and corresponding compilation rules defined. The computer environment has also evolved in parallel, which has subsequently allowed the storage of larger amount of information and various new functionalities. One important aim of the NRDC community has always been to provide sufficient description of the experimental conditions in order to allow proper data interpretation. Evaluations of the quality and consistency of the compilation of the following types of data have been recently conducted.

\subsection{Neutron resonance data from TOF facilities}

A Consultants' Meeting on "EXFOR data in Resonance Region and Spectrometer's Response Function" [11] was organised by NDS to discuss the compilation of the time-of-flight (TOF) measurements in order to allow proper interpretation and evaluation of TOF data. In particular, the storage of the response functions of the measurement set-ups was discussed. A template formulated during the workshop was recommended to be used by compilers to collect and compile experimental details. Compilation guidelines ensure good consistency in the compilation of all types of data in EXFOR. Following the meeting, the collaborations between nuclear data centres and TOF facilities led to amendments of the data in resonance regions. The n_TOF Collaboration has also established a procedure for data submission through the data dissemination coordinator, who has a strong background in EXFOR compilation. Collection, submission and incorporation into EXFOR of all data measured at the $\mathrm{n}_{-} \mathrm{TOF}$ facility are currently underway as a joint effort with $n_{-}$TOF, NDS and NEA-DB staff [12]. A major improvement in the EXFOR availability of the energy-dependent data measured at the ORELA facility has also been achieved due to cooperation between NNDC and Guber (ORNL). Recovery efforts have led to the collection and inclusion of F, Al, Si, Cl, Ti, Fe, Mn, Cr, $\mathrm{NI}, \mathrm{K}, \mathrm{Sn}, \mathrm{Nd}$, and U capture, transmission and fission data in EXFOR.

\subsection{Thermal neutron scattering data}

Renewed interest in the revision of existing thermalscattering libraries and the undertaking of further evaluations driven by new applications necessitated a thorough inspection of EXFOR for neutron-induced data at thermal energies. Considering the physics of thermal scattering and differences in the analyses of scattering at epithermal and higher energies, agreement was reached that a review of the compilation rules for thermal-scattering data was required. Furthermore, as well as the isotopic composition, the compound, structure, phase, orientation, thermodynamic and other physical properties have to be considered. A Consultants' Meeting on "Compilation of Thermal Neutron Scattering Data in EXFOR" was organised by NDS in 2015 [13]. Various experimental and analytical techniques applied at thermal energies were discussed. The description of the thermal scattering quantities in the EXFOR compiler's manual (LEXFOR) has been revised. Recommendations to compilers and guidelines concerning the compilation of experimental information were issued. Only nuclear quantities (double-differential cross-section, differential cross-section, and total cross-section) should be compiled in EXFOR. NDS staff were advised to set up a supplementary database for the $S(q, \omega), S(\alpha, \beta)$ phonon spectrum, structural parameters etc. to support and facilitate thermal neutron scattering data analyses and evaluations. About 120 datasets have been compiled and made available on the meeting website. NDS also received a collection of publications that were missing from EXFOR for assessment, assignment and compilation within the NRDC network.

\subsection{Verification and validation for neutron-induced threshold reaction cross-sections}

A comprehensive review of the cross-section data for neutron-induced threshold reactions has been carried out by NEA-DB staff. The review combines statistical analysis of the deviations of the EXFOR-compiled data with the corresponding values in the major evaluated nuclear data libraries, and verification of the correctness of the compiled data against the original publications those cases with large deviations. About 25000 reactions have been included in the statistical analysis, from which about 10000 have been subject to a more detailed review. A description of the method is included in the report, together with the outcome of the statistical analysis for the different types of reaction within each evaluated data library and graphical comparison of the experimental data and evaluations for a number of reactions [14]. This review is part of the WPEC Subgroup 30 ("Quality improvement of the EXFOR database") follow-up activities led by NEA-DB.

\subsection{Definitions of radioisotope thick target yields}

The experimental thick target yield data are important for the development of radioisotope production in medical applications and validation of the excitation functions. However, there are inconsistencies in the expressions of the thick target yield quantities in the literature (e.g., for some 
authors the unit $1 \mu \mathrm{A}-\mathrm{h}$ means irradiation by projectiles corresponding to a total charge of $1 \mu \mathrm{A} \times 1 \mathrm{~h}=3.6 \mathrm{mC}$, while other authors consider $1 \mu \mathrm{A}-\mathrm{h}$ as an irradiation at $1 \mu \mathrm{A}$ beam current for $1 \mathrm{~h}$ ). A review of the measurement technique, definitions and recommendations on reporting thick target yield data have been published with the aims of ensuring consistency and valid inter-comparisons of the published data from different experiments [15]. Authors are advised to provide thick target yield data in $\mathrm{MBq} / \mathrm{C}$ (and additionally in $\mathrm{MBq} / \mu \mathrm{A}-\mathrm{h}$ if necessary), and end-of-bombardment and saturation thick target yields in $\mathrm{MBq} / \mu \mathrm{A}$.

\subsection{Half-lives adopted in decay analysis}

The half-lives adopted by experimentalists for their decay analyses of the reaction products should be recorded in EXFOR as information for cross-section evaluators. NDS compared half-lives coded in EXFOR in 2014 with those in Nuclear Wallet Cards [16]. About 1180 halflives were found to deviate by more than $50 \%$ from the half-lives recommended in Nuclear Wallet Cards. All such cases were checked against the values given in the publications. The compilation mistakes and incorrect half-lives, confirmed by authors, were corrected. A better consistency in the isomeric state assignment with the Nuclear Wallet Cards data is now provided. For example the $5 \mathrm{hr}$ level of ${ }^{110} \mathrm{In}$ is coded as a ground state in all EXFOR entries even though it is treated as a metastable state in some old articles.

\subsection{Duplication}

As a rule an EXFOR entry represents the results of work performed at a given laboratory at a given time. If the same experimental work is partly published in different scientific publications all of them have to be included in one entry with the most representative paper considered as the main reference. However, if not stated clearly by the authors or overlooked by the compiler, two or more entries may be identified with the same experimental results. This cannot just be considered a technical problem because such an approach may eventually give a higher weighting factor to a particular experiment in an evaluation. Plots of both preliminary and final data sets by EXFOR users must be avoided. Both preliminary and final data sets are systematically compiled in EXFOR for intermediate energy charged-particle induced reaction activation crosssections measured by the group led by Michel (Leibniz Universität Hannover) and neutron induced reaction activation cross-sections measured at the OKTAVIAN facility by the group led by Kawade (Nagoya University). Efforts to identify pairs of data sets from the same experiment are not always trivial exercises, but we have systematically improved the situation by taking advice from the authors in order to define such data in EXFOR.

\section{Summary and conclusions}

The International Network of Nuclear Reaction Data Centres continues to collaborate in the collection, compilation and dissemination of experimental nuclear reaction data. New articles continue to be compiled in a timely manner. Collaboration with authors is extremely important to ensure availability of the original experimental data and compilation of the key experimental information. There have been no major amendments to either the scope of the EXFOR database or the format. Recently our efforts have focused mainly on improving the completeness and quality of the compilation for specific data types that are important for the development of nuclear physics and applications.

\section{References}

[1] N. Otuka et al., Nucl. Data Sheets 120, 272 (2014)

[2] N. Otuka and M. Herman (eds.), INDC(NDS)-0718 (2016), IAEA, Vienna, Austria

[3] S.F. Mughabghab, Atlas of Neutron Resonances, Elsevier Science (2006)

[4] E.J. Axton, GE/PH/01/86, CBNM (1986)

[5] N. Otuka et al., in these proceedings

[6] S. Simakov et al., EPJ Web of Conf. 106, 04011 (2016)

[7] Y. Xu et al., Nucl. Phys. A 918, 61 (2013)

[8] N.E. Holden et al., BNL-NCS-35513-R, BNL (1984), BNL-NCS-36379, BNL (1985)

[9] M.B. Chadwick et al., these proceedings

[10] P.J. Griffin et al. (eds.), INDC(NDS)-0719 (2016), IAEA, Vienna, Austria

[11] F. Gunsing, P. Schillebeeckx, V. Semkova (eds.), INDC(NDS)-0647 (2013), IAEA, Vienna, Austria

[12] E. Dupont et al., in these proceedings

[13] J.I. Marquez Damian, V. Semkova (eds.), INDC(NDS)-0697 (2016), IAEA, Vienna, Austria

[14] A. Koning, NEA/DB/DOC(2014)3 (2014), NEA Data Bank, Paris, France

[15] N. Otuka, S. Takács, Radiochim. Acta 103, 1 (2015)

[16] J.K. Tuli, Nuclear Wallet Cards, BNL (2011) 